\title{
Graph-Theoretic Approach to Exponential Stability of Delayed Coupled Systems on Networks under Periodically Intermittent Control
}

\author{
Beibei Guo, Yu Xiao and Chiping Zhang \\ Department of Mathematics, Harbin Institute of Technology \\ 150001 Harbin, China \\ E-mail(corresp.): yxiao@hit.edu.cn
}

Received March 21, 2017; revised December 9, 2017; accepted December 10, 2017

\begin{abstract}
In this paper, the exponential stability of delayed coupled systems on networks (DCSNs) is investigated via periodically intermittent control. By utilizing graph-theoretic approach and Lyapunov function method, a novel method for stability analysis of DCSNs is developed. Moreover, some useful and easily verifiable sufficient conditions are presented in the form of Lyapunov-type theorem and coefficients-type criterion. These laws reveal that the stability has a close relationship with the topology structure of the networks. In addition, as a subsequent result, the obtained theory is successfully applied to study the exponential stability of delayed coupled oscillators on networks under periodically intermittent control. Finally, a numerical example is given to validate the effectiveness of theoretical results.
\end{abstract}

Keywords: delayed coupled systems, periodically intermittent control, graph-theoretic method, exponential stability.

AMS Subject Classification: 94C15; 34H05; 37B25.

\section{Introduction}

During the past decades, networks have gained great attentions owing to their widespread applications in communication networks, electricity supply networks, engineering and many other areas $[11,13,23,29]$. It has been observed that many complex networks with intricate interactions among their components are described by coupled systems on networks (CSNs), such as neural networks, coupled biological systems, ecological systems and so on. As is well known, stability is one main property of CSNs, and plays a vital role in their 
extensive applications in theory and practice. For instance, if a network is employed to solve some optimization problems, it is highly desirable for the network to have a unique globally stable equilibrium. On the other hand, time delay unavoidably exists in many practical systems and is thus naturally considered in CSNs. As shown in [1], the existence of time delay could make delayed CSNs be oscillation or instability. Therefore, the stability analysis of CSNs with time delay have recently been studied by many researchers for better understanding about the collective behavior of a wide variety of physical, chemical, and biological problems $[3,9,24,33]$. A large amount of sufficient conditions have been obtained to determine the stability of delayed systems.

Recently, many control technique have been proposed to deal with the various issues of networks, such as event-triggered control [12], feedback control [31], charging control [14], intermittent control and so on. Among them, intermittent control has gained considerable research attention due to its wide applications in many fields, such as manufacturing, transportation and communication $[5,26,36]$. A significant benefit of intermittent control is that it has a nonzero control width, and can be easily implemented in practice. For example, in communications, when the strength of the system signal is below the required level, the external control signal can be added to achieve the desired result or requirement. Motivated by this practical consideration, intermittent control has been successfully applied to stabilize and synchronize nonlinear dynamical systems [8], neural networks [28], chaotic systems [10], and even complex networks [34]. The stability and synchronization analysis problems for general CSNs under intermittent control has received increasing research interest and many relevant results have been reported in the literature (see e.g. [27, 32, 34] and the references therein). Therefore, it is significant and practical to study the stability of delayed coupled systems on networks (DCSNs) by using the intermittent control technique.

Up to now, searching the common Lyapunov functions or variations of the same framework is the main tool for studying dynamic characteristics of a system. However, in terms of the intricate relations between the topological structure and divergent dynamical properties, it is quite tough to construct a proper Lyapunov function for large-scale system. Fortunately, with the help of Kirchhoff's Matrix Tree Theorem in graph theory, Li et al. [6,15] presented a systematic method to construct a global Lyapunov function. They obtained some criteria to guarantee the global stability and applied the theoretical results to coupled oscillators, epidemic models and predator-prey models. Following this pioneering work, numerous results have been reported. For instance, in [16], the authors studied the global stability of coupled nonlinear systems with Markovian switching by graph theory; in [35], Zhang et al. considered the existence and global exponential stability of periodic solution for a neutral coupled systems based on graph-theoretic method; in [17], Li et al. used Razumikhin technique and graph theory to investigate the exponential stability of delayed multi-group model with reaction-diffusion and multiple dispersal. Additionally, this technique has also been successfully applied in stochastic CSs [20], time delay CSs [4], discrete CSs [2], impulse CSs [22] and multi-diffusion CSs [21] and so on. Inspired by the pioneering work, DCSNs can be represented by a 
directed graph. However, as far as we know, there are few scholars considering the stability of DCSNs by adopting graph-theoretic approach and periodically intermittent control technique.

In this paper, we make first attempt to investigate exponential stability of DCSNs under periodically intermittent control. Based on graph theory and Lyapunov function method, two types of sufficient criteria are proposed, of which one is given in the form of Lyapunov functions and network topology, while the other is given by the means of coefficients in system, which is more convenient to be tested in practice since it mainly depends on the coefficients of considered system. Furthermore, as the applications of theoretical results, a delayed coupled oscillators on network under periodically intermittent control is also considered. At the same time, some stability criteria for it are presented. Finally, a numerical example is given to illustrate the effectiveness of the proposed method in this paper.

Differing from the previous works on the stability of DCSNs, the key contributions of this paper lie in the following. First, a periodically intermittent control scheme is used to consider exponential stability of DCSNs. Second, we analyze the exponential stability of DCSNs under periodically intermittent control by means of combining graph theory with Lyapunov function method. Some results in graph theory are effectively utilized to avoid the difficulty in directly finding the global Lyapunov function of DCSNs. Third, the theoretical results are applied to delayed coupled oscillators on network with periodically intermittent control.

The rest of this paper is organized as follows. The model formulation is given in Section 2. In Section 3, some sufficient criteria of exponential stability for DCSNs under periodically intermittent control are arranged. In Section 4, the stability results under periodically intermittent control for delayed coupled oscillators on networks are presented. Section 5 provides a numerical example to certify the effectiveness of the proposed theory. Finally, we use a conclusion to close the paper.

\section{Model formulation}

In this section, we first introduce some useful notations. Basic concepts on graph theory in this paper can be found in the Appendix. Then, we present the model formulation considered in this paper and finally give a kind of stability definition and an important lemma in graph theory.

The following notations will be used throughout this paper. Let $\mathbb{R}$ be the set of all real numbers, $\mathbb{R}^{n}$ be the $n$-dimensional Euclidean space, $\mathbb{R}_{+}^{1}$ be the set of all nonnegative real numbers and $\mathbb{N}^{+}=\{0,1,2, \ldots\}$. Also, let $\ell=\{1,2, \ldots, l\}, \mathbb{Z}^{+}=\{1,2, \ldots\}, m=\sum_{i=1}^{l} m_{i}$ for $m_{i} \in \mathbb{Z}^{+}$and $|\cdot|$ be the Euclidean norm in $\mathbb{R}^{n}$. The superscript ' $\mathrm{T}^{\prime}$ stands for the transpose. In addition, $C^{1,1}\left(\mathbb{R}^{n} \times \mathbb{R}_{+}^{1} ; \mathbb{R}_{+}^{1}\right)$ represents for the family of all nonnegative functions $V(x, t)$ on $\mathbb{R}^{n} \times \mathbb{R}_{+}^{1}$ which are continuously differentiable in $x$ and $t$, and $C\left([-\tau, 0] ; \mathbb{R}^{n}\right)$ is the space of continuous functions $x:[-\tau, 0] \rightarrow \mathbb{R}^{n}$ with norm $\|x\|=\sup _{-\tau \leq t \leq 0}|x(t)|$.

Now, we give the model formulation considered in this paper. Based on 
graph theory, a network can be represented by a digraph $\mathcal{G}$ with $l(l \geq 2)$ vertices. As alluded to in the Introduction, time delay is important in large-scale networks. Note that the existence of delay may cause bad performance, e.g. instability and oscillation. It leads to considerable technical complications, but this generality would be natural in practice. In addition, compared with continuous control methods, intermittent control is more effective. For these reasons, we will study the stability problem of DCSNs under periodically intermittent control, the $k$-th vertex system is constructed as follows:

$$
\begin{aligned}
\dot{x}_{k}(t)=b_{k}\left(x_{k}(t)\right)+\sum_{h=1}^{l} c_{k h} f_{h}\left(x_{h}(t)\right) & +\sum_{h=1}^{l} d_{k h} g_{h}\left(x_{h}\left(t-\tau_{k h}\right)\right) \\
& +u_{k}(t), \quad t \geq 0, \quad k \in \ell
\end{aligned}
$$

where $x_{k}(t)$ corresponds to the state of the $k$-th vertex at time $t ; b_{k}(\cdot): \mathbb{R}^{m_{k}} \rightarrow$ $\mathbb{R}^{m_{k}}$ is an appropriate behaved function; $f_{h}(\cdot), g_{h}(\cdot): \mathbb{R}^{m_{h}} \rightarrow \mathbb{R}^{m_{k}}$ denotes the inner connecting functions in each vertex; $0 \leq \tau_{k h}$ is the discrete time delay along $h$-th vertex system from $k$-th vertex system; the notations $c_{k h}, d_{k h}$ are the strength of the coupling and the time delay connection weight, respectively. If there is a connection from vertex $k$ to vertex $h$, then the coupling $c_{k h} \neq 0$, $d_{k h} \neq 0$; otherwise, $c_{k h}=0, d_{k h}=0 ; u_{k}(t)$ is an intermittent control defined by

$$
u_{k}(t)=\left\{\begin{array}{lr}
\sum_{h=1}^{l} Y_{k h} x_{h}(t), & n T \leq t<(n+\theta) T, \\
0, & (n+\theta) T \leq t<(n+1) T,
\end{array}\right.
$$

where $n \in \mathbb{N}^{+}, Y_{k h}(k, h \in \ell)$ are the constant control, $T$ is the control period, $0<\theta<1$ is called the rate of control duration. Here we assume that system (2.1) is supplemented with initial value given by

$$
x(t)=\phi(t), \quad t \in[-\tau, 0],
$$

$\phi(t)=\left(\phi_{1}^{\mathrm{T}}(t), \phi_{2}^{\mathrm{T}}(t), \ldots, \phi_{l}^{\mathrm{T}}(t)\right)^{\mathrm{T}} \in C\left([-\tau, 0], \mathbb{R}^{m}\right)$ and $\tau=\max _{k, h \in \ell}\left\{\tau_{k h}\right\}$. Furthermore, let $b_{k}(0)=0, f_{h}(0)=0$ and $g_{h}(0)=0$, then, the system (2.1) admits a trivial solution $x(t)=\left(x_{1}^{\mathrm{T}}(t), x_{2}^{\mathrm{T}}(t), \ldots, x_{l}^{\mathrm{T}}(t)\right)^{\mathrm{T}}=(0,0, \ldots, 0)^{\mathrm{T}}=0$.

A definition on the exponential stability of the trivial solution and a useful lemma in graph theory are given as follows.

Definition 1. [8] Suppose that there exist constants $M \geq 1$ and $\lambda>0$ satisfying

$$
|x(t)| \leq M\|\phi\| e^{-\lambda t}, \quad t \geq 0,
$$

then the trivial solution of system (2.1) is exponentially stable.

Lemma 1. [15] Suppose that $l \geq 2$. Let $c_{k}$ denote the cofactor of the $k$-th diagonal element of $L$. Then the following identity holds:

$$
\sum_{k, h=1}^{l} c_{k} \delta_{k h} F_{k h}\left(x_{k}, x_{h}\right)=\sum_{\mathcal{Q} \in \mathbb{Q}} W(\mathcal{Q}) \sum_{(s, r) \in E\left(C_{\mathcal{Q}}\right)} F_{r s}\left(x_{r}, x_{s}\right),
$$


where for any $k, h \in \ell, F_{k h}\left(x_{k}, x_{h}\right)$ is an arbitrary function, $\mathbb{Q}$ is the set of all spanning unicyclic graphs of $(\mathcal{G}, A), W(\mathcal{Q})$ is the weight of $\mathcal{Q}$, and $C_{\mathcal{Q}}$ denotes the directed cycle of $\mathcal{Q}$. Particularly, if $(\mathcal{G}, A)$ is strongly connected, then $c_{k}>0$ for $k \in \ell$.

\section{Stability Analysis for DCSNs}

In this section, with the help of graph theory and Lyapunov function method as well as periodically intermittent control, two kinds of exponential stability criteria for DCSNs are established in the form of Lyapunov function and the coefficients of DCSNs. Moreover, the proofs of our main results are given in details. Let us first establish a Lyapunov-type theorem.

\subsection{Lyapunov-type theorem}

In order to further study, we need to prepare a definition about $k$-th vertexLyapunov function following [30] and two basic assumptions first.

Definition 2. For $k, h \in \ell$ and $p \geq 2$, let the following conditions hold:

A1. There exist positive constants $\alpha_{k}$ and $\beta_{k}$, such that

$$
\alpha_{k}\left|x_{k}(t)\right|^{p} \leq V_{k}\left(x_{k}(t), t\right) \leq \beta_{k}\left|x_{k}(t)\right|^{p} .
$$

A2. There exist constants $\sigma_{k}>0, \sigma_{k}^{1}>0, \delta_{k h}>0, k, h \in \ell$ and function $M_{k h}\left(X_{k}, X_{h}\right) \in C\left(\mathbb{R}^{p m_{k}} \times \mathbb{R}^{p m_{h}} ; \mathbb{R}\right)$, such that

$$
\frac{\mathrm{d} V_{k}\left(x_{k}(t), t\right)}{\mathrm{d} t} \leq\left\{\begin{array}{c}
-\sigma_{k}\left|x_{k}(t)\right|^{p}+\sum_{h=1}^{l} \delta_{k h}\left|x_{k}\left(t-\tau_{h k}\right)\right|^{p}+\sum_{h=1}^{l} \delta_{k h} M_{k h}\left(X_{k}, X_{h}\right), \\
n T \leq t<(n+\theta) T, \\
-\sigma_{k}^{1}\left|x_{k}(t)\right|^{p}+\sum_{h=1}^{l} \delta_{k h}\left|x_{k}\left(t-\tau_{h k}\right)\right|^{p}+\sum_{h=1}^{l} \delta_{k h} M_{k h}\left(X_{k}, X_{h}\right), \\
(n+\theta) T \leq t<(n+1) T,
\end{array}\right.
$$

where $\sigma_{k}=\sigma_{k}^{1}-p Y_{k k}-(p-1) \sum_{h=1, h \neq k}^{l}\left|Y_{k h}\right|, X_{k}=\left|x_{k}(t)\right|^{p}+\left|x_{k}\left(t-\tau_{h k}\right)\right|^{p}$ and $X_{h}=\left|x_{h}(t)\right|^{p}+\left|x_{h}\left(t-\tau_{k h}\right)\right|^{p}$.

A3. Along each directed cycle $\mathcal{C}$ of weighted digraph $(\mathcal{G}, A)$, there is

$$
\sum_{(h, k) \in E(\mathcal{C})} M_{k h}\left(X_{k}, X_{h}\right) \leq 0
$$

for all $X_{k} \in \mathbb{R}^{p m_{k}}, X_{h} \in \mathbb{R}^{p m_{h}}$. Then function $V_{k}\left(x_{k}(t), t\right) \in C^{1,1}\left(\mathbb{R}^{m_{k}} \times \mathbb{R}_{+}^{1} ; \mathbb{R}_{+}^{1}\right)$ is called the $k$-th vertex-Lyapunov function for system (2.1).

Assumption 1. For $k \in \ell$, system (2.1) admits vertex-Lyapunov function $V_{k}\left(x_{k}(t), t\right)$, the following condition holds,

$$
\min _{k \in \ell}\left\{\sigma_{k} / \beta_{k}\right\}>\max _{k \in \ell}\left\{\sum_{h=1}^{l} \delta_{k h} / \alpha_{k}\right\} .
$$


It follows from Assumption 1 that there exists a sufficiently small $\varepsilon>0$, such that

$$
-\varepsilon+\min _{k \in \ell}\left\{\sigma_{k} / \beta_{k}\right\}-\max _{k \in \ell}\left\{\sum_{h=1}^{l} \delta_{k h} / \alpha_{k}\right\} e^{\varepsilon \tau}>0 .
$$

Assumption 2. $\varepsilon-\zeta(1-\theta)>0$, where $\zeta=\max _{k \in \ell}\left\{\left|\varsigma_{k} / \beta_{k}\right|\right\}$ and $\varsigma_{k}=p Y_{k k}+$ $(p-1) \sum_{h=1, h \neq k}^{l}\left|Y_{k h}\right|$.

Remark 1. Conditions A1 and A2 show that the decay of $p$-th moment of the solution is exponential, which are applicable to the exponential stability of coupled systems in some applications. It can be seen from condition A2 that $\delta_{k h} \geq 0$, so $\delta_{k h}$ can not be sufficiently large to ensure $\dot{V}_{k}\left(x_{k}(t), t\right) \leq 0$. Hence, Assumption 1 provides a restriction for weighted matrix $\left(\delta_{k h}\right)_{l \times l}$ to guarantee the stability of DCSNs.

Remark 2. Assumption 2 provides a way to compute the rate of control duration $\theta$, namely $\theta>1-\varepsilon / \zeta$, if the parameter $\varepsilon, \beta_{k}$ and control constant $Y_{k h}$ are properly chosen.

We now begin to establish the main result in this subsection to guarantee exponential stability of system (2.1).

Theorem 1. Let digraph $(\mathcal{G}, A)$ be strongly connected, in which $A=\left(\delta_{k h}\right)_{l \times l}$. Suppose that the system (2.1) admits the vertex-Lyapunov function $V_{k}\left(x_{k}, t\right)$ for any $k \in \ell$, and Assumptions 1 and 2 hold, then the trivial solution of system (2.1) is exponentially stable under periodically intermittent control (2.2).

Proof. First, we set

$$
V(x, t)=\sum_{k=1}^{l} c_{k} V_{k}\left(x_{k}(t), t\right),
$$

where $c_{k}$ is the cofactor of the $k$-th diagonal element of the Laplacian matrix of $(\mathcal{G}, A)$. Noting that $(\mathcal{G}, A)$ is strongly connected, we have $c_{k}>0$ for any $k \in \ell$ by Lemma 1 . Denote $\beta=\sum_{k=1}^{l} c_{k} \beta_{k}$ and

$$
\alpha=\left(\sum_{k=1}^{l} c_{k} \alpha_{k}\right)^{1-p / 2}\left(\min _{k \in \ell}\left\{c_{k} \alpha_{k}\right\}\right)^{p / 2} .
$$

Thus, from (3.1) and (3.4) we have

$$
V(x, t) \leq \sum_{k=1}^{l} c_{k} \beta_{k}\left|x_{k}(t)\right|^{p} \leq\left(\sum_{k=1}^{l} c_{k} \beta_{k}\right)|x(t)|^{p},
$$

and

$$
V(x, t) \geq \sum_{k=1}^{l} c_{k} \alpha_{k}\left|x_{k}(t)\right|^{p}=\sum_{i=1}^{l} c_{i} \alpha_{i}
$$




$$
\begin{aligned}
& \times \sum_{k=1}^{l}\left[\frac{c_{k} \alpha_{k}}{\sum_{j=1}^{l} c_{j} \alpha_{j}}\left(\left|x_{k}(t)\right|^{2}\right)^{\frac{p}{2}}\right] \geq \sum_{i=1}^{l} c_{i} \alpha_{i}\left[\sum_{k=1}^{l} \frac{c_{k} \alpha_{k}}{\sum_{j=1}^{l} c_{j} \alpha_{j}}\left|x_{k}(t)\right|^{2}\right]^{\frac{p}{2}} \\
& \geq\left(\sum_{k=1}^{l} c_{k} \alpha_{k}\right)^{1-\frac{p}{2}}\left(\min _{k \in \ell}\left\{c_{k} \alpha_{k}\right\}\right)^{\frac{p}{2}}|x(t)|^{p}=\alpha|x(t)|^{p}
\end{aligned}
$$

Hence,

$$
\alpha|x|^{p} \leq V(x, t) \leq \beta|x|^{p} .
$$

For simplicity, now we divide the proof into the following three steps.

Step 1. Prove $P(t)=e^{\varepsilon t} V(x, t)-h \beta\|\phi\|^{p}<0$, for all $t \in[-\tau, \theta T)$, where $h>1$ is a constant.

It is easy to verify that, for all $t \in[-\tau, 0], P(t)<0$. Next, we prove that for $t \in[0, \theta T)$ :

$$
P(t)<0 \text {. }
$$

Otherwise, there exists a $t_{0} \in[0, \theta T)$ such that

$$
\begin{aligned}
& P\left(t_{0}\right)=0, \quad \mathrm{D}^{+} P\left(t_{0}\right) \geq 0, \\
& P(t)<0, \quad \text { for } t \in\left[0, t_{0}\right),
\end{aligned}
$$

where $\mathrm{D}^{+}$denotes Dini derivative. Making use of Lemma 1, condition $A 3$ and a fact $W(\mathcal{Q})>0$, it yields

$$
\sum_{k, h=1}^{l} c_{k} \delta_{k h} M_{k h}\left(X_{k}, X_{h}\right)=\sum_{\mathcal{Q} \in \mathbb{Q}} W(\mathcal{Q}) \sum_{(s, r) \in E\left(C_{\mathcal{Q}}\right)} M_{r s}\left(X_{r}, X_{s}\right) \leq 0 .
$$

Then, applying the conditions A1-A2, (3.4), and (3.7)-(3.9), we can derive that

$$
\begin{aligned}
\mathrm{D}^{+} & P\left(t_{0}\right)=\varepsilon e^{\varepsilon t_{0}} V\left(x\left(t_{0}\right), t_{0}\right)+e^{\varepsilon t_{0}} \dot{V}\left(x\left(t_{0}\right), t_{0}\right) \\
\leq & e^{\varepsilon t_{0}}\left\{\varepsilon V\left(x\left(t_{0}\right), t_{0}\right)-\sum_{k=1}^{l} c_{k} \sigma_{k}\left|x_{k}\left(t_{0}\right)\right|^{p}+\sum_{k, h=1}^{l} c_{k} \delta_{k h}\left|x_{k}\left(t_{0}-\tau_{h k}\right)\right|^{p}\right\} \\
& +e^{\varepsilon t_{0}}\left\{\sum_{k, h=1}^{l} c_{k} \delta_{k h} M_{k h}\left(X_{k}, X_{h}\right)\right\} \\
\leq & e^{\varepsilon t_{0}}\left[\varepsilon V\left(x\left(t_{0}\right), t_{0}\right)-\min _{k \in \ell}\left\{\sigma_{k} / \beta_{k}\right\} V\left(x\left(t_{0}\right), t_{0}\right)\right. \\
& \left.+\max _{k \in \ell}\left\{\sum_{h=1}^{l} \delta_{k h} / \alpha_{k}\right\} V\left(x_{k}\left(t_{0}-\tau_{h k}\right), t_{0}-\tau_{h k}\right)\right] \\
< & \left(\varepsilon-\min _{k \in \ell}\left\{\sigma_{k} / \beta_{k}\right\}\right) h \beta\|\phi\|^{p}+\max _{k \in \ell}\left\{\sum_{h=1}^{l} \delta_{k h} / \alpha_{k}\right\} e^{\varepsilon \tau} h \beta\|\phi\|^{p} \\
= & -\left(-\varepsilon+\min _{k \in \ell}\left\{\sigma_{k} / \beta_{k}\right\}-\max _{k \in \ell}\left\{\sum_{h=1}^{l} \delta_{k h} / \alpha_{k}\right\} e^{\varepsilon \tau}\right) h \beta\|\phi\|^{p} .
\end{aligned}
$$


From (3.3), it implies $\mathrm{D}^{+} P\left(t_{0}\right)<0$, which leads to a contradiction with $\mathrm{D}^{+} P\left(t_{0}\right) \geq 0$. Hence inequality (3.6) holds.

Step 2. Prove $Q(t)=e^{\varepsilon t} V(x, t)-h \beta\|\phi\|^{p} e^{\zeta(t-\theta T)}<0$, for all $t \in[\theta T, T)$.

Otherwise, there exists a $t_{1} \in[\theta T, T)$ such that

$$
Q\left(t_{1}\right)=0, \quad \mathrm{D}^{+} Q\left(t_{1}\right) \geq 0, \quad Q(t)<0, \quad \text { for } t \in\left[\theta T, t_{1}\right) .
$$

For $k, h \in \ell$, if $\theta T \leq t_{1}-\tau_{h k}<t_{1}$, we get

$$
e^{\varepsilon t_{1}} \sum_{k=1}^{l} c_{k} V_{k}\left(x_{k}\left(t_{1}-\tau_{h k}\right), t_{1}-\tau_{h k}\right)<e^{\varepsilon \tau} h \beta\|\phi\|^{p} e^{\zeta\left(t_{1}-\theta T\right)}
$$

and if $-\tau \leq t_{1}-\tau_{h k}<\theta T$, from Step 1, we have

$$
e^{\varepsilon t_{1}} \sum_{k=1}^{l} c_{k} V_{k}\left(x_{k}\left(t_{1}-\tau_{h k}\right), t_{1}-\tau_{h k}\right)<e^{\varepsilon \tau} h \beta\|\phi\|^{p}<e^{\varepsilon \tau} h \beta\|\phi\|^{p} e^{\zeta\left(t_{1}-\theta T\right)} .
$$

Hence, for any $k \in \ell$, we always have

$$
e^{\varepsilon t_{1}} \sum_{k=1}^{l} c_{k} V_{k}\left(x_{k}\left(t_{1}-\tau_{h k}\right), t_{1}-\tau_{h k}\right)<e^{\varepsilon \tau} h \beta\|\phi\|^{p} e^{\zeta\left(t_{1}-\theta T\right)} .
$$

Then,

$$
\begin{aligned}
\mathrm{D}^{+} Q & \left(t_{1}\right)=\varepsilon e^{\varepsilon t_{1}} V\left(x\left(t_{1}\right), t_{1}\right)+e^{\varepsilon t_{1}} \dot{V}\left(x\left(t_{1}\right), t_{1}\right)-\zeta h \beta\|\phi\|^{p} e^{\zeta\left(t_{1}-\theta T\right)} \\
\leq & e^{\varepsilon t_{1}}\left\{\varepsilon V\left(x\left(t_{1}\right), t_{1}\right)-\sum_{k=1}^{l} c_{k} \sigma_{k}^{1}\left|x_{k}\left(t_{1}\right)\right|^{p}+\sum_{k, h=1}^{l} c_{k} \delta_{k h}\left|x_{k}\left(t_{1}-\tau_{h k}\right)\right|^{p}\right\} \\
& -\zeta h \beta\|\phi\|^{p} e^{\zeta\left(t_{1}-\theta T\right)}+e^{\varepsilon t_{1}}\left\{\sum_{k, h=1}^{l} c_{k} \delta_{k h} M_{k h}\left(X_{k}, X_{h}\right)\right\} \\
< & \left(\varepsilon-\min _{k \in \ell}\left\{\sigma_{k}^{1} / \beta_{k}\right\}\right) h \beta\|\phi\|^{p} e^{\zeta\left(t_{1}-\theta T\right)} \\
& +\max _{k \in \ell}\left\{\sum_{h=1}^{l} \delta_{k h} / \alpha_{k}\right\} e^{\varepsilon \tau} h \beta\|\phi\|^{p} e^{\zeta\left(t_{1}-\theta T\right)}-\zeta h \beta\|\phi\|^{p} e^{\zeta\left(t_{1}-\theta T\right)} \\
= & -\left(-\varepsilon+\min _{k \in \ell}\left\{\sigma_{k} / \beta_{k}\right\}-\max _{k \in \ell}\left\{\sum_{h=1}^{l} \delta_{k h} / \alpha_{k}\right\} e^{\varepsilon \tau}\right) h \beta\|\phi\|^{p} e^{\zeta\left(t_{1}-\theta T\right)} \\
& -\frac{\varsigma_{k}}{\beta_{k}} h \beta\|\phi\|^{p} e^{\zeta\left(t_{1}-\theta T\right)}-\zeta h \beta\|\phi\|^{p} e^{\zeta\left(t_{1}-\theta T\right)} .
\end{aligned}
$$

According to (3.3) and Assumption 2, we have $\mathrm{D}^{+} Q\left(t_{1}\right)<0$, which contradicts $\mathrm{D}^{+} Q\left(t_{1}\right) \geq 0$. Hence $Q(t)<0$ holds for all $t \in[\theta T, T)$.

From Step 1 we get that, for $t \in[-\tau, \theta T)$,

$$
e^{\varepsilon t} V(x, t)<h \beta\|\phi\|^{p}<h \beta\|\phi\|^{p} e^{\zeta(1-\theta) T} .
$$


It follows from Step 2, for $t \in[\theta T, T)$, we have

$$
e^{\varepsilon t} V(x, t)<h \beta\|\phi\|^{p} e^{\zeta(t-\theta T)}<h \beta\|\phi\|^{p} e^{\zeta(1-\theta) T} .
$$

Thus, for all $t \in[-\tau, T)$, the following inequality holds,

$$
e^{\varepsilon t} V(x, t)<h \beta\|\phi\|^{p} e^{\zeta(1-\theta) T} .
$$

Similar to the proof of (3.6), we can get that

$$
e^{\varepsilon t} V(x, t)<h \beta\|\phi\|^{p} e^{\zeta(1-\theta) T},
$$

for $T \leq t<(1+\theta) T$. Analogous to the proof of Step 2 , one sees that

$$
e^{\varepsilon t} V(x, t)<h \beta\|\phi\|^{p} e^{\zeta(1-\theta) T} e^{\zeta[t-(1+\theta) T]}=h \beta\|\phi\|^{p} e^{\zeta(t-2 \theta T)},
$$

for $(1+\theta) T \leq t<2 T$.

Step 3. Applying mathematical induction method, we can certificate the following estimates are true. For $n T \leq t<(n+\theta) T$,

$$
e^{\varepsilon t} V(x, t)<h \beta\|\phi\|^{p} e^{n \zeta(1-\theta) T}
$$

and for $(n+\theta) T \leq t<(n+1) T$,

$$
e^{\varepsilon t} V(x, t)<h \beta\|\phi\|^{p} e^{n \zeta(1-\theta) T} e^{\zeta[t-(n+\theta) T]}=h \beta\|\phi\|^{p} e^{\zeta[t-(n+1) \theta T]} .
$$

The proofs of (3.10) and (3.11) are similar to those of [8], therefore we omit this part to avoid redundancy. Thus, it follows from mathematical induction method that inequalities (3.10) and (3.11) hold for any integer $n>0$. For any $t \geq 0$, there exists a natural integer $n$ such that $n T \leq t<(n+\theta) T$. When $n T \leq t<(n+\theta) T$, and $n \leq t / T$, we have

$$
e^{\varepsilon t} V(x, t)<h \beta\|\phi\|^{p} e^{n \zeta(1-\theta) T} \leq h \beta\|\phi\|^{p} e^{\zeta(1-\theta) t} .
$$

Moreover, when $(n+\theta) T \leq t<(n+1) T$ and $n+1>t / T$, we get

$$
e^{\varepsilon t} V(x, t)<h \beta\|\phi\|^{p} e^{\zeta[t-(n+1) \theta T]}<h \beta\|\phi\|^{p} e^{\zeta(1-\theta) t} .
$$

Finally, let $h \rightarrow 1$, according to (3.5), it is readily seen that

$$
e^{\varepsilon t} \alpha|x(t)|^{p} \leq e^{\varepsilon t} V(x, t) \leq \beta\|\phi\|^{p} e^{\zeta(1-\theta) t} .
$$

Hence, we get the following estimate

$$
|x(t)| \leq(\beta / \alpha)^{\frac{1}{p}}\|\phi\| e^{-\frac{1}{p}[\varepsilon-\zeta(1-\theta)] t}=M\|\phi\| e^{-\lambda t},
$$

for any $t \geq 0$, where $\lambda=\frac{\varepsilon-\zeta(1-\theta)}{p}, M=\left(\frac{\beta}{\alpha}\right)^{\frac{1}{p}}$. It follows from $\alpha \leq \beta$ and Definition 1 that the trivial solution of system (2.1) is exponentially stable. The proof is therefore complete. 
Remark 3. The feature of this paper is that the CSNs is described by a digraph (see Figure 1), in which each vertex of digraph stands for an individual system called vertex system, and the inter-connections and interactions among vertex systems are characterized by the directed arcs of digraph. Hence, based on the graph-theoretic approach, a new method is presented by utilizing the vertexLyapunov function $V_{k}$, which is first applied to analyze the exponential stability of DCSNs under periodically intermittent control. What is more, the sufficient conditions obtained in Theorem 1 show that exponential stability of DCSNs has a close relation with topological structure of CSs. The validity of the technique is presented in Theorem 2.

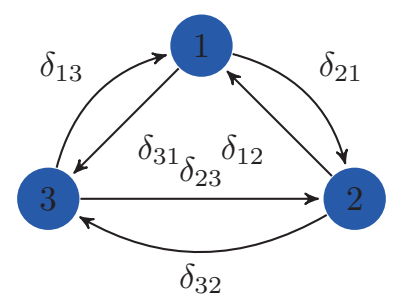

Figure 1. A digraph $\mathcal{G}$ with 3 vertices.

Remark 4. It is well-known that the method of Lyapunov function is general for the investigation of stability for systems. Actually, it is a challenge to construct Lyapunov function for high dimensional systems directly. It is worth pointing that, by using some results in graph theory, a global Lyapunov function $V$ of DCSNs is successfully constructed via the vertex-Lyapunov functions $V_{k}$ and the topology property of systems. That is, $V(x, t)=\sum_{k=1}^{l} c_{k} V_{k}\left(x_{k}, t\right)$, where $c_{k}$ is the cofactor of the $k$-th diagonal element of the Laplacian matrix of $(\mathcal{G}, A)$. Thus, this avoids to directly seek for the Lyapunov function of DCSNs. That means, with the help of graph theory, CSNs can be studied much more easily.

Remark 5. It should be pointed out that condition A3 holds for each directed cycle $\mathcal{C}$ and any $X_{k}$ and $X_{h}$. Since the number of directed cycles may be very large and $X_{k}, X_{h}$ are arbitrary, we must check A3 for infinite times. It seems to be so complicated. However, this problem can be successfully solved if we find some appropriate functions $M_{k h}\left(X_{h}, X_{k}\right), k, h=1,2, \ldots l$. We shall now take one further step to study some others simple and easy-checked conditions based on some results in graph theory.

Note that if $(\mathcal{G}, A)$ is balanced, we can readily get

$$
\sum_{k, h=1}^{l} c_{k} \delta_{k h} M_{k h}\left(X_{k}, X_{h}\right)=\frac{1}{2} \sum_{\mathcal{Q} \in \mathbb{Q}} W(\mathcal{Q}) \sum_{(h, k) \in E\left(C_{\mathcal{Q}}\right)}\left[M_{k h}\left(X_{k}, X_{h}\right)+M_{h k}\left(X_{h}, X_{k}\right)\right] .
$$


Thus, (3.2) can be replaced by

$$
\sum_{(h, k) \in E\left(C_{\mathcal{Q}}\right)}\left[M_{k h}\left(X_{k}, X_{h}\right)+M_{h k}\left(X_{h}, X_{k}\right)\right] \leq 0 .
$$

Based on this, a corollary is obtained as follows.

Corollary 1. Suppose that $(\mathcal{G}, A)$ is balanced, then the conclusion of Theorem 1 holds if (3.2) is replaced by (3.12).

Furthermore, in view that if for every $M_{k h}\left(X_{k}, X_{h}\right)$ there exist functions $P_{k}\left(X_{k}\right)$ and $P_{h}\left(X_{h}\right)$, such that

$$
M_{k h}\left(X_{k}, X_{h}\right) \leq P_{k}\left(X_{k}\right)-P_{h}\left(X_{h}\right),
$$

then (3.2) can be readily verified. We thus obtain one more corollary below.

Corollary 2. The conclusion of Theorem 1 holds if (3.2) is replaced by (3.13).

In the discussions above, a Lyapunov-type theorem is presented in the form of Lyapunov functions and topological structure. In what follows, we explore that how to find the detailed functions $M_{k h}\left(X_{k}, X_{h}\right)$ to check the availability of Theorem 1, which is the task of Theorem 2 .

\subsection{Coefficients-type criterion}

In this subsection, a coefficients-type criterion is presented as follows.

Theorem 2. Suppose that the following conditions hold:

B1. There exist positive constants $\eta_{k}, A_{h}, B_{h}$, such that

$$
\begin{aligned}
& x_{k}^{\mathrm{T}} b_{k}\left(x_{k}\right) \leq-\eta_{k}\left|x_{k}\right|^{2}, \\
& \left|f_{h}\left(x_{h}\right)\right| \leq A_{h}\left|x_{h}\right|, \\
& \left|g_{h}\left(x_{h}\right)\right| \leq B_{h}\left|x_{h}\right| .
\end{aligned}
$$

B2. Digraph $(\mathcal{G}, A)$ is strongly connected and the following inequality holds:

$$
\min _{k \in l}\left\{p \eta_{k}-\left|c_{k k}\right| A_{k}-J_{k}-\zeta_{k}-\sum_{h=1}^{l} \delta_{k h}\right\}>\max _{k \in l}\left\{\sum_{h=1}^{l} \delta_{k h}\right\},
$$

where $J_{k}=(p-1)\left(\sum_{h=1}^{l}\left|c_{k h}\right| A_{h}+\sum_{h=1}^{l}\left|d_{k h}\right| B_{h}\right), A=\left(\delta_{k h}\right)_{l \times l}, p \geq 2$ and

$$
\delta_{k h} \triangleq \begin{cases}\max \left\{\left|c_{k h}\right| A_{h}+\left|Y_{k h}\right|,\left|d_{k h}\right| B_{h}\right\}, & h \neq k, \\ 0, & h=k .\end{cases}
$$

Then the trivial solution of system (2.1) is exponentially stable.

Proof. For any $k \in \ell$, denote $V_{k}\left(x_{k}(t), t\right)=\left|x_{k}(t)\right|^{p}$, which satisfies condition A1 obviously. In the sequel, computing the time derivative of $V_{k}\left(x_{k}(t), t\right)$ along the trajectories of system (2.1). When $n T \leq t<(n+\theta) T$, we can get

$$
\frac{\mathrm{d} V_{k}\left(x_{k}(t), t\right)}{\mathrm{d} t}=p\left|x_{k}(t)\right|^{p-2} x_{k}^{\mathrm{T}}(t) \dot{x}_{k}(t)
$$




$$
\begin{aligned}
& \leq p\left|x_{k}(t)\right|^{p-2} x_{k}^{\mathrm{T}}(t) b_{k}\left(x_{k}(t)\right)+p\left|x_{k}(t)\right|^{p-2}\left|x_{k}^{\mathrm{T}}(t)\right| \sum_{h=1}^{l}\left|c_{k h}\right|\left|f_{h}\left(x_{h}(t)\right)\right| \\
& +p\left|x_{k}(t)\right|^{p-2} x_{k}^{\mathrm{T}}(t) Y_{k k} x_{k}(t)+p\left|x_{k}(t)\right|^{p-2}\left|x_{k}^{\mathrm{T}}(t)\right| \sum_{h=1, h \neq k}^{l}\left|Y_{k h}\right|\left|x_{h}(t)\right| \\
& +p\left|x_{k}(t)\right|^{p-2}\left|x_{k}^{\mathrm{T}}(t)\right| \sum_{h=1}^{l}\left|d_{k h}\right|\left|g_{h}\left(x_{h}\left(t-\tau_{k h}\right)\right)\right| .
\end{aligned}
$$

Applying the following inequality (see [18], p. 52)

$$
|a|^{p}|b|^{q} \leq \frac{p}{p+q}|a|^{p+q}+\frac{q}{p+q}|b|^{p+q}
$$

it yields

$$
\begin{aligned}
& p\left|x_{k}(t)\right|^{p-2}\left|x_{k}^{\mathrm{T}}(t)\right| \sum_{h=1, h \neq k}^{l}\left|Y_{k h}\right|\left|x_{h}(t)\right| \leq p\left|x_{k}(t)\right|^{p-1} \sum_{h=1, h \neq k}^{l}\left|Y_{k h}\right|\left|x_{h}(t)\right| \\
& \leq(p-1) \sum_{h=1, h \neq k}^{l}\left|Y_{k h}\right|\left|x_{k}(t)\right|^{p}+\sum_{h=1, h \neq k}^{l}\left|Y_{k h}\right|\left|x_{h}(t)\right|^{p} .
\end{aligned}
$$

Together with (3.15) and (3.18), we have

$$
\begin{aligned}
& p\left|x_{k}(t)\right|^{p-2}\left|x_{k}^{\mathrm{T}}(t)\right| \sum_{h=1}^{l}\left|c_{k h}\right|\left|f_{h}\left(x_{h}(t)\right)\right| \leq p\left|x_{k}(t)\right|^{p-1} \sum_{h=1}^{l}\left|c_{k h}\right| A_{h}\left|x_{h}(t)\right| \\
& \leq(p-1) \sum_{h=1}^{l}\left|c_{k h}\right| A_{h}\left|x_{k}(t)\right|^{p}+\left|c_{k k}\right| A_{k}\left|x_{k}(t)\right|^{p}+\sum_{h=1, h \neq k}^{l}\left|c_{k h}\right| A_{h}\left|x_{h}(t)\right|^{p} .
\end{aligned}
$$

From (3.16) and (3.18), one obtains

$$
\begin{aligned}
& p\left|x_{k}(t)\right|^{p-2}\left|x_{k}^{\mathrm{T}}(t)\right| \sum_{h=1}^{l}\left|d_{k h}\right|\left|g_{h}\left(x_{h}\left(t-\tau_{k h}\right)\right)\right| \leq p\left|x_{k}(t)\right|^{p-1} \sum_{h=1}^{l}\left|d_{k h}\right| B_{h}\left|x_{h}\left(t-\tau_{k h}\right)\right| \\
& \quad=p \sum_{h=1}^{l}\left|d_{k h}\right| B_{h}\left|x_{k}(t)\right|^{p-1}\left|x_{h}\left(t-\tau_{k h}\right)\right| \\
& \quad \leq(p-1) \sum_{h=1}^{l}\left|d_{k h}\right| B_{h}\left|x_{k}(t)\right|^{p}+\sum_{h=1}^{l}\left|d_{k h}\right| B_{h}\left|x_{h}\left(t-\tau_{k h}\right)\right|^{p} .
\end{aligned}
$$

Submitting (3.14) and (3.19)-(3.21) into (3.17), one sees that

$$
\begin{gathered}
\frac{\mathrm{d} V_{k}\left(x_{k}, t\right)}{\mathrm{d} t} \leq\left(-\eta_{k} p+\left|c_{k k}\right| A_{k}+(p-1) \sum_{h=1}^{l}\left|c_{k h}\right| A_{h}+(p-1) \sum_{h=1}^{l}\left|d_{k h}\right| B_{h}\right. \\
\left.\quad+p Y_{k k}+(p-1) \sum_{h=1, h \neq k}^{l}\left|Y_{k h}\right|\right)\left|x_{k}(t)\right|^{p}+\left(\sum_{h=1, h \neq k}^{l}\left|c_{k h}\right| A_{h}\right.
\end{gathered}
$$




$$
\begin{aligned}
& \left.+\sum_{h=1, h \neq k}^{l}\left|Y_{k h}\right|\right)\left|x_{h}(t)\right|^{p}+\sum_{h=1}^{l}\left|d_{k h}\right| B_{h}\left|x_{h}\left(t-\tau_{k h}\right)\right|^{p} \\
\leq & -\left(\eta_{k} p-\left|c_{k k}\right| A_{k}-(p-1) \sum_{h=1}^{l}\left|c_{k h}\right| A_{h}-(p-1) \sum_{h=1}^{l}\left|d_{k h}\right| B_{h}-\varsigma_{k}\right. \\
& \left.-\sum_{h=1}^{l} \delta_{k h}\right)\left|x_{k}(t)\right|^{p}-\sum_{h=1}^{l} \delta_{k h}\left|x_{k}(t)\right|^{p}+\sum_{h=1}^{l} \delta_{k h}\left|x_{h}(t)\right|^{p} \\
& +\sum_{h=1}^{l} \delta_{k h}\left|x_{h}\left(t-\tau_{k h}\right)\right|^{p}-\sum_{h=1}^{l} \delta_{k h}\left|x_{k}\left(t-\tau_{h k}\right)\right|^{p}+\sum_{h=1}^{l} \delta_{k h}\left|x_{k}\left(t-\tau_{h k}\right)\right|^{p} \\
= & -\sigma_{k}\left|x_{k}(t)\right|^{p}+\sum_{h=1}^{l} \delta_{k h}\left|x_{k}\left(t-\tau_{h k}\right)\right|^{p}+\sum_{h=1}^{l} \delta_{k h}\left[\left|x_{h}(t)\right|^{p}+\left|x_{h}\left(t-\tau_{k h}\right)\right|^{p}\right. \\
& \left.-\left(\left|x_{k}(t)\right|^{p}+\left|x_{k}\left(t-\tau_{h k}\right)\right|^{p}\right)\right] \\
= & -\sigma_{k}\left|x_{k}(t)\right|^{p}+\sum_{h=1}^{l} \delta_{k h}\left|x_{k}\left(t-\tau_{h k}\right)\right|^{p}+\sum_{h=1}^{l} \delta_{k h} M_{k h}\left(X_{k}, X_{h}\right),
\end{aligned}
$$

in which

$$
\begin{aligned}
& \sigma_{k}=\eta_{k} p-\left|c_{k k}\right| A_{k}-(p-1) \sum_{h=1}^{l}\left|c_{k h}\right| A_{h}-(p-1) \sum_{h=1}^{l}\left|d_{k h}\right| B_{h}-\varsigma_{k}-\sum_{h=1}^{l} \delta_{k h}, \\
& M_{k h}\left(X_{k}, X_{h}\right)=\left(\left|x_{h}(t)\right|^{p}+\left|x_{h}\left(t-\tau_{k h}\right)\right|^{p}\right)-\left(\left|x_{k}(t)\right|^{p}+\left|x_{k}\left(t-\tau_{h k}\right)\right|^{p}\right) .
\end{aligned}
$$

Similarly, when $(n+\theta) T \leq t<(n+1) T$, we can get

$$
\begin{aligned}
& \frac{\mathrm{d} V_{k}\left(x_{k}(t), t\right)}{\mathrm{d} t} \leq-\left(\eta_{k} p-\left|c_{k k}\right| A_{k}-(p-1) \sum_{h=1}^{l}\left|c_{k h}\right| A_{h}-(p-1) \sum_{h=1}^{l}\left|d_{k h}\right| B_{h}\right. \\
& \left.\quad-\sum_{h=1}^{l} \delta_{k h}\right)\left|x_{k}(t)\right|^{p}-\sum_{h=1}^{l} \delta_{k h}\left|x_{k}(t)\right|^{p}+\sum_{h=1}^{l} \delta_{k h}\left|x_{h}(t)\right|^{p} \\
& \quad+\sum_{h=1}^{l} \delta_{k h}\left|x_{h}\left(t-\tau_{k h}\right)\right|^{p}-\sum_{h=1}^{l} \delta_{k h}\left|x_{k}\left(t-\tau_{h k}\right)\right|^{p}+\sum_{h=1}^{l} \delta_{k h}\left|\left(x_{k}\left(t-\tau_{h k}\right)\right)\right|^{p} \\
& =-\sigma_{k}^{1}\left|x_{k}(t)\right|^{p}+\sum_{h=1}^{l} \delta_{k h}\left|x_{k}\left(t-\tau_{h k}\right)\right|^{p}+\sum_{h=1}^{l} \delta_{k h} M_{k h}\left(X_{k}, X_{h}\right),
\end{aligned}
$$

in which

$$
\sigma_{k}^{1}=\eta_{k} p-\left|c_{k k}\right| A_{k}-(p-1) \sum_{h=1}^{l}\left|c_{k h}\right| A_{h}-(p-1) \sum_{h=1}^{l}\left|d_{k h}\right| B_{h}-\sum_{h=1}^{l} \delta_{k h} .
$$

Hence, condition A2 holds. Moreover, it is easy to verify that all functions $M_{k h}$ satisfy (3.2). Thus, the trivial solution of system (2.1) is exponentially stable by Theorem 1 and condition B2. This completes the proof. 
Remark 6. An important feature of the result in Theorem 2 is that stability criteria for system (2.1) are obtained according to the coefficients of system. Contrasting with Theorem 1 discussed previously, the stability conditions in Theorem 2 are easier to be checked. In what follows, under periodically intermittent control, we shall use an example of delayed coupled oscillators on networks to illustrate the availability of above results.

\section{An application to delayed coupled oscillators on net- works}

In this section, we will discuss the exponential stability of delayed coupled oscillators on networks (DCONS) under periodically intermittent control. The following nonlinear oscillation is one of the commonest examples in the broad applications in physics, mechanics, electrical engineering and so on.

$$
x^{\prime \prime}(t)+\varphi(x(t)) x^{\prime}(t)+x(t)=0,
$$

in which $t \geq 0, x(t) \in \mathbb{R}$ is the state variable; $\varphi(x) \geq 0$ is the damping coefficient. Many properties of system (4.1) such as boundedness, stability and limit cycles, have been studied widely $[7,19]$. Here nonlinear oscillator network with time-varying delay on a diagraph $\mathcal{G}$ with $l(l \geq 2)$ vertices, for the $k$-th vertex it is assigned a time delay oscillator described by

$x_{k}^{\prime \prime}(t)+\varphi_{k}\left(x_{k}(t)\right) x_{k}^{\prime}(t)+x_{k}(t)+\sum_{h=1}^{l} v_{k h} N_{h}\left(x_{h}(t)\right)+\sum_{h=1}^{l} b_{k h} H_{h}\left(x_{h}\left(t-\tau_{k h}\right)\right)=0$,

where $k \in \ell, x_{k}(t) \in \mathbb{R}, N_{h}, H_{h}: \mathbb{R} \rightarrow \mathbb{R}$ is the form of influence from vertex $h$ to vertex $k$. $v_{k h}, b_{k h}$ represents the intensity of influence from vertex $h$ to vertex $k$. If there is a connection from vertex $k$ to vertex $h$, then the coupling $v_{k h} \neq 0, b_{k h} \neq 0$; otherwise, $v_{k h}=0, b_{k h}=0$.

As is well-known, the system may be unstable in the process of selecting the parameters or time delay. Thus, in the following, we use periodically intermittent control to stabilize the system (4.2). Let $y_{k}(t)=x_{k}^{\prime}(t)+\zeta_{k} x_{k}(t)$, we can get that

$$
\left\{\begin{aligned}
x_{k}^{\prime}(t) & =y_{k}(t)-\zeta_{k} x_{k}(t)+u_{k}(t) \\
y_{k}^{\prime}(t) & =\left(\zeta_{k}-\varphi_{k}\left(x_{k}(t)\right)\right) y_{k}(t)-x_{k}(t)-\sum_{h=1}^{l} v_{k h} N_{h}\left(x_{h}(t)\right) \\
& -\sum_{h=1}^{l} b_{k h} H_{h}\left(x_{h}\left(t-\tau_{k h}\right)\right)+\left(\zeta_{k} \varphi_{k}\left(x_{k}(t)\right)-\zeta_{k}^{2}\right) x_{k}(t),
\end{aligned}\right.
$$

where $u_{k}(t)$ is periodically intermittent control defined by (2.2).

The following theorem is provided to guarantee exponential stability of the trivial solution of system (4.2).

Theorem 3. Let digraph $(\mathcal{G}, \bar{D})$ be strongly connected. The trivial solution of system (4.2) is exponentially stable provided that the following conditions are satisfied. 
C1. For any $k \in \ell$, there are positive constants $m_{k}, M_{k} \geq m_{k}$ and $\gamma_{k}$, $\epsilon_{k}$ such that

$$
m_{k} \leq \varphi_{k}\left(x_{k}\right) \leq M_{k},\left|N_{h}\left(x_{h}\right)\right| \leq \gamma_{h}\left|x_{h}\right|,\left|H_{h}\left(x_{h}\right)\right| \leq \epsilon_{h}\left|x_{h}\right| .
$$

C2. There exist positive constants $p \geq 2$ and $\mu_{k}$, such that $\mu_{k} \leq m_{k},\left(2 M_{k}-\right.$ $\left.\mu_{k}\right) \mu_{k} \leq 4$ and

$$
\min _{k \in l}\left\{p \mu_{k}-4\left|v_{k k}\right| \gamma_{k}-\bar{J}_{k}-4 \zeta_{k}-4 \sum_{h=1}^{l} \delta_{k h}^{1}\right\}>\max _{k \in l}\left\{4 \sum_{h=1}^{l} \delta_{k h}^{1}\right\},
$$

where $\bar{J}_{k}=4(p-1)\left(\sum_{h=1}^{l}\left|v_{k h}\right| \gamma_{h}+\sum_{h=1}^{l}\left|b_{k h}\right| \epsilon_{h}\right), \bar{D}=\left(\delta_{k h}^{1}\right)_{l \times l}$ and

$$
\delta_{k h}^{1} \triangleq \begin{cases}\max \left\{\left|v_{k h}\right| \gamma_{h}+\left|Y_{k h}\right|,\left|b_{k h}\right| \epsilon_{h}\right\}, & h \neq k \\ 0, & h=k\end{cases}
$$

Proof. First of all, denote vector-valued functions $X_{k}(t)=\left(x_{k}(t), y_{k}(t)\right)^{\mathrm{T}}$ and

$$
F_{k}\left(X_{k}(t), t\right)=\left(\begin{array}{c}
y_{k}(t)-\zeta_{k} x_{k}(t) \\
\left(\zeta_{k}-\varphi_{k}\left(x_{k}(t)\right)\right) y_{k}(t)+\left(\zeta_{k} \varphi_{k}\left(x_{k}(t)\right)-\zeta_{k}^{2}-1\right) x_{k}(t)
\end{array}\right) .
$$

By simple calculation, we can get

$$
\begin{aligned}
X_{k}^{\mathrm{T}}(t) & F_{k}\left(X_{k}(t), t\right) \\
\leq & \left|\zeta_{k} \varphi_{k}\left(x_{k}(t)\right)-\zeta_{k}^{2}\right|\left|x_{k}(t) y_{k}(t)\right|-\zeta_{k} x_{k}^{2}(t)+\left(\zeta_{k}-\varphi_{k}\left(x_{k}(t)\right)\right) y_{k}^{2}(t) \\
\leq & -\left(\zeta_{k}-\frac{\delta_{k}^{2}}{2}\left(\zeta_{k} \varphi_{k}\left(x_{k}(t)\right)-\zeta_{k}^{2}\right)\right) x_{k}^{2}(t) \\
& -\left(\varphi_{k}\left(x_{k}(t)\right)-\zeta_{k}-\frac{1}{2 \delta_{k}^{2}}\left(\zeta_{k} \varphi_{k}\left(x_{k}(t)\right)-\zeta_{k}^{2}\right)\right) y_{k}^{2}(t),
\end{aligned}
$$

where $\delta_{k}$ is an arbitrary value, then let $\delta_{k}^{2}=\zeta_{k}=\frac{\mu_{k}}{2}$, then we have

$$
X_{k}^{\mathrm{T}}(t) F_{k}\left(X_{k}(t), t\right) \leq-\frac{\mu_{k}}{4}\left|X_{k}\right|^{2}
$$

So condition B1 is satisfied. On the other hand,

$$
\begin{aligned}
& \min _{k \in l}\left\{p \eta_{k}-\left|c_{k h}\right| A_{h}-J_{k}-\zeta_{k}-\sum_{h=1}^{l} \delta_{k h}\right\}-\max _{k \in l}\left\{\sum_{h=1}^{l} \delta_{k h}\right\} \\
= & \min _{k \in l}\left\{p \frac{\mu_{k}}{4}-\left|v_{k h}\right| \gamma_{h}-\bar{J}_{k}-\zeta_{k}-\sum_{h=1}^{l} \delta_{k h}^{1}\right\}-\max _{k \in l}\left\{\sum_{h=1}^{l} \delta_{k h}^{1}\right\},
\end{aligned}
$$

where $\eta_{k}=\mu_{k} / 4, A_{h}=\gamma_{h}, B_{h}=\epsilon_{h}$. Therefore, condition B2 is fulfilled. Hence, the trivial solution of system (4.2) is exponentially stable according to Theorem 2. This completes the proof. 


\section{Numerical test}

A detailed numerical example is provided in this section to demonstrate the practical applicability of the theoretical results.

Example: Let $l=4$. We proceed to consider the delayed coupled oscillators on networks (4.2).

Assume that $\varphi_{k}\left(x_{k}\right)=0.25-0.2 \sin x_{k}, N_{h}\left(x_{h}\right)=0.1 x_{h}, H_{h}\left(x_{h}\right)=x_{h}$ and some parameters are presented as follows: $p=2, \tau=1, \zeta_{1}=0.01, \zeta_{2}=0.03$, $\zeta_{3}=0.05, \zeta_{4}=0.04$. The other parameters of system (4.2) are given in Table 1.

Table 1. The values of $v_{k h}$ and $b_{k h}$

\begin{tabular}{ccccc}
\hline$v_{k h}$ & 1 & 2 & 3 & 4 \\
\hline 1 & 0 & 0.04 & 0 & -0.09 \\
2 & 0.03 & 0 & 0 & 0.05 \\
3 & 0 & -0.2 & 0 & 0.08 \\
4 & 0.07 & 0 & 0.01 & 0 \\
\hline
\end{tabular}

\begin{tabular}{lcccc}
\hline$b_{k h}$ & 1 & 2 & 3 & 4 \\
\hline 1 & 0 & 0.01 & 0 & 0.05 \\
2 & 0 & 0 & 0.02 & 0.01 \\
3 & 0 & -0.05 & 0 & 0.02 \\
4 & 0.05 & -0.06 & 0.01 & 0 \\
\hline
\end{tabular}

The weighted matrix of digraph $(\mathcal{G}, \bar{D})$ is also given as follows

$$
\left(\delta_{k h}^{1}\right)_{4 \times 4}=\left(\begin{array}{cccc}
0.000 & 0.010 & 0.000 & 0.050 \\
0.003 & 0.000 & 0.020 & 0.010 \\
0.000 & 0.020 & 0.000 & 0.020 \\
0.050 & 0.060 & 0.010 & 0.000
\end{array}\right) .
$$

By simple calculations, we can get $\mu_{1}=0.02, \mu_{2}=0.06, \mu_{3}=0.1, \mu_{4}=0.08$, $\gamma_{h}=0.1, \epsilon_{h}=1$ for $k, h=1,2,3,4$. Choosing $Y_{11}=-0.3, Y_{22}=-0.3$, $Y_{33}=-0.25, Y_{44}=-0.4, Y_{k h}=0(k, h=1,2,3,4, k \neq h)$. Then $\zeta=0.8$ and $\varepsilon=0.072$ by computation. From Assumption 2 that $\theta>0.91$. Here we select $\theta=0.92$ and $T=5$. The conditions $\mathrm{C} 1$ and $\mathrm{C} 2$ in Theorem 3 are all fulfilled, and thus system (4.2) is exponentially stable.

Furthermore, the initial values associated with system (4.2) are given in (5.1). The numerical simulation results are presented in Figures 2 and 3.

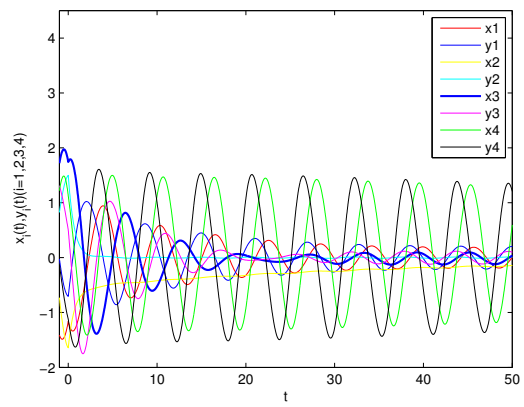

Figure 2. Simple paths of the solution to system (4.2) with initial values (5.1) 


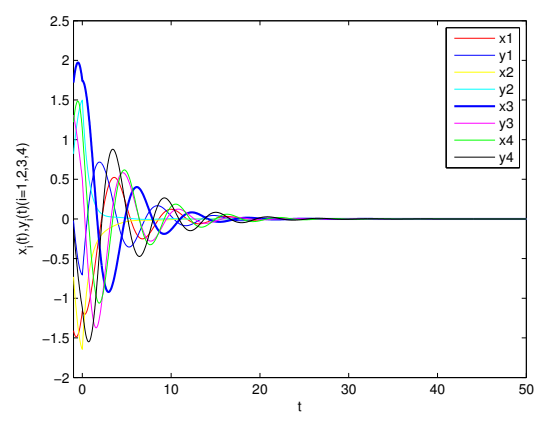

Figure 3. Simple path of the solution to system (4.3) under periodically intermittent control with initial values $(5.1)$

Figure 2 is the paths of the solution to system (4.2) without the control. Figure 3 is the respond of the solution to system (4.3) under periodically intermittent control. We can clearly see from presented figures that periodically intermittent control plays a critical role in the exponential stability of system (4.3). In fact, these simulation results support our theoretical results:

$$
\begin{aligned}
& x_{1}(t)=-1.4 \cos t-0.5 \sin t, \quad y_{1}(t)=-0.92 \sin t-0.2 \cos 2 t \sin t, \\
& x_{2}(t)=-1.5 \sin t-0.7 \cos t, \quad y_{2}(t)=0.6 \sin t+0.5 \cos t, \quad t \in[-1,0] \\
& x_{3}(t)=\sin t+1.7 \cos t, \quad y_{3}(t)=0.82 \cos t-0.5 \sin t+0.5, \\
& x_{4}(t)=0.5 \sin t+0.6 \cos t+0.4, \quad y_{4}(t)=-1.2 \sin t-0.1 .
\end{aligned}
$$

\section{Acknowledgements}

The authors are very grateful to the reviewers for carefully reading the paper and for their valuable comments and suggestions which have improved the paper.

\section{References}

[1] P. Baldi and A. Atiya. How delays affect neural dynamics and learning. IEEE Trans. Neural Netw., 5(4):612-621, 1994. https://doi.org/10.1109/72.298231.

[2] T. Boukhobza and F. Hamelin. Discrete mode observability of structured switching descriptor linear systems: a graph-theoretic approach. Automatica, 49(10):3042-3048, 2013. https://doi.org/10.1016/j.automatica.2013.06.006.

[3] J. Cao, P. Li and W. Wang. Global synchronization in arrays of delayed neural networks with constant and delayed coupling. Phys. Lett. A, 353(4):318-325, 2006. https://doi.org/10.1016/j.physleta.2005.12.092.

[4] H. Chen and J. Sun. Stability analysis for coupled systems with time delay on networks. Physica A, 391(3):528-534, 2012. https://doi.org/10.1016/j.physa.2011.08.037. 
[5] K. Cuomo and A. Oppenheim. Circuit implementation of synchronized chaos with applications to communications. Phys. Rev. Lett., 71(1):65-68, 1993. https://doi.org/10.1103/PhysRevLett.71.65.

[6] H. Guo, M. Y. Li and Z. Shuai. A graph-theoretic approach to the method of global Lyapunov functions. P. Am. Math. Soc., 136:2793-2802, 2008. https://doi.org/10.1090/S0002-9939-08-09341-6.

[7] J. Heagy, T. Carroll and L. Pecora. Synchronous chaos in coupled oscillator systems. Phys. Rev. E, 50(3):1874-1885, 1994. https://doi.org/10.1103/PhysRevE.50.1874.

[8] C. Hu, J. Yu, H. Jiang and Z. Teng. Exponential lag synchronization for neural networks with mixed delays via periodically intermittent control. Chaos, 20(2):023108, 2010. https://doi.org/10.1063/1.3391900.

[9] T. Huang, C. Li, S. Duan and J. Starzyk. Robust exponential stability of uncertain delayed neural networks with stochastic perturbation and impulse effects. IEEE Trans. Neural Netw. Learn. Syst., 23(6):866-875, 2012. https://doi.org/10.1109/TNNLS.2012.2192135.

[10] C. Li, X. Liao and T. Huang. Exponential stabilization of chaotic systems with delay by periodically intermittent control. Chaos, 17(1):013103, 2007. https://doi.org/10.1063/1.2430394.

[11] C. Li, X. Yu, T. Huang and X. He. Distributed optimal consensus over resource allocation network and its application to dynamical economic dispatch. IEEE Trans. Neural Netw. Learn. Syst., PP(99):1-12, 2017. https://doi.org/10.1109/TNNLS.2017.2691760.

[12] H. Li, G. Chen, T. Huang and Z. Dong. High-performance consensus control in networked systems with limited bandwidth communication and time-varying directed topologies. IEEE Trans. Neural Netw. Learn. Syst., 28(5):1043-1054, 2017. https://doi.org/10.1109/TNNLS.2016.2519894.

[13] H. Li, G. Chen, T. Huang and Z. Dong et al. Event-triggered distributed average consensus over directed digital networks with limited communication bandwidth. IEEE T. Cybern., 46(12):3098-3110, 2016. https://doi.org/10.1109/TCYB.2015.2496977.

[14] J. Li, C. Li, Y. Xu and Z. Dong et al. Noncooperative gamebased distributed charging control for plug-in electric vehicles in distribution networks. IEEE Trans. Ind. Inform., $\mathbf{P P}(99): 1-1,2016$. https://doi.org/10.1109/TII.2016.2632761.

[15] M.Y. Li and Z. Shuai. Global-stability problem for coupled systems of differential equations on networks. J. Differ. Equ., 248(1):1-20, 2010. https://doi.org/10.1016/j.jde.2009.09.003.

[16] W. Li, H. Su, D. Wei and K. Wang. Global stability analysis of coupled nonlinear systems with Markovian switching. Commun. Nonlinear Sci. Numer. Simulat., 17(6):2609-2616, 2012. https://doi.org/10.1016/j.cnsns.2011.09.039.

[17] W. Li, X. Zhang and C. Zhang. Exponential stability of delayed multi-group model with reaction-diffusion and multiple dispersal based on Razumikhin technique and graph theory. Commun. Nonlinear Sci. Numer. Simulat., 27(1-3):237253, 2015. https://doi.org/10.1016/j.cnsns.2015.03.012.

[18] X. Mao and C. Yuan. Stochastic differential equations with Markovian switching. Imperial College Press, 2006. https://doi.org/10.1142/p473. 
[19] D. Reddy, A. Sen and G. Johnston. Time delay induced death in coupled limit cycle oscillators. Phys. Rev. Lett., 80(23):5109-5112., 1998. https://doi.org/10.1103/PhysRevLett.80.5109.

[20] H. Su, W. Li, K. Wang and X. Ding. Stability analysis for stochastic neural network with infinite delay. Neurocomputing, 74(10):1535-1540, 2011. https://doi.org/10.1016/j.neucom.2010.12.027.

[21] H. Su, P. Wang and X. Ding. Stability analysis of discrete-time coupled systems with multi-diffusion by graph-theoretic approach and its applications. Discrete Contin. Dyn. Syst-Ser. B, 21:253-269, 2016.

[22] J. Suo, J. Sun and Y. Zhang. Stability analysis for impulsive coupled systems on networks. Neurocomputing, 99:172-177, 2013. https://doi.org/10.1016/j.neucom.2012.06.002.

[23] P. Venkataram, S. Ghosal and B.P.V. Kumar. Neural network based optimal routing algorithm for communication networks. Neural Netw., 15(10):1289-1298, 2002. https://doi.org/10.1016/S0893-6080(02)00067-9.

[24] Z. Wang, Z. Duan and J. Cao. Impulsive synchronization of coupled dynamical networks with nonidentical duffing oscillators and coupling delays. Chaos, 22(1):013140, 2012. https://doi.org/10.1063/1.3692971.

[25] D. West. Introduction to graph theory. Prentice Hall, Upper Saddle River, 1996.

[26] J. Xiao, G. Hu and Z. Qu. Synchronization of spatiotemporal chaos and its application to multichannel spread-spectrum communication. Phys. Rev. Lett., 77(20):4162-4165, 1996. https://doi.org/10.1103/PhysRevLett.77.4162.

[27] X. Yang and J. Cao. Stochastic synchronization of coupled neural networks with intermittent control. Phys. Lett. A, 373(36):3259-3272, 2009. https://doi.org/10.1016/j.physleta.2009.07.013.

[28] J. Yu, C. Hu and H. Jiang. Exponential synchronization of Cohen-Grossberg neural networks via periodically intermittent control. Neurocomputing, 74(10):17761782, 2011. https://doi.org/10.1016/j.neucom.2011.02.015.

[29] C. Zhang, W. Li and K. Wang. Boundedness for network of stochastic coupled van der Pol oscillators with time-varying delayed coupling. Appl. Math. Model., 37(7):5394-5402, 2013. https://doi.org/10.1016/j.apm.2012.10.032.

[30] C. Zhang, W. Li and K. Wang. A graph-theoretic approach to stability of neutral stochastic coupled oscillators network with time-varying delayed coupling. Math. Meth. Appl. Sci., 37(8):1179-1190, 2014. https://doi.org/10.1002/mma.2879.

[31] C. Zhang, W. Li and K. Wang. Graph-theoretic method on exponential synchronization of stochastic coupled networks with Markovian switching. Nonlinear Anal.-Hybrid Syst., 15:37-51, 2015. https://doi.org/10.1016/j.nahs.2014.07.003.

[32] G. Zhang and Y. Shen. Exponential stability of memristor-based chaotic neural networks with time-varying delays via intermittent control. IEEE Trans. Neural Netw. Learn. Syst., 26(7):1431-1441, 2015. https://doi.org/10.1109/TNNLS.2014.2345125.

[33] J. Zhang and X. Guo. Stability and bifurcation analysis in the delaycoupled van der Pol oscillators. Appl. Math. Model., 34(9):2291-2299, 2010. https://doi.org/10.1016/j.apm.2009.10.037. 
[34] W. Zhang, C. Li, T. Huang and J. Huang. Stability and synchronization of memristor-based coupling neural networks with time-varying delays via intermittent control. Neurocomputing, 173(Part 3):1066-1072, 2016. https://doi.org/10.1016/j.neucom.2015.08.063.

[35] X. Zhang, W. Li and K. Wang. The existence and global exponential stability of periodic solution for a neutral coupled system on networks with delays. Appl. Math. Comput., 264:208-217, 2015. https://doi.org/10.1016/j.amc.2015.04.109.

[36] C. Zhou and T. Chen. Digital communication robust to transmission error via chaotic synchronization based on contraction maps. Phys. Rev. Lett., 56(2):15991604, 1997. https://doi.org/10.1103/PhysRevE.56.1599.

\section{Appendix}

To analyze the stability for DCSNs over directed network topology, we now state some basic concepts of graph theory. For detail, we refer the reader to $[15,25]$. A digraph $\mathcal{G}=(\ell, E)$ contains a set $\ell$ of vertices and a set $E$ of $\operatorname{arcs}(i, j)$ leading from initial vertex $i$ to terminal vertex $j$. A subgraph $\mathcal{H}$ of $\mathcal{G}$ is said to be spanning if $\mathcal{H}$ and $\mathcal{G}$ have the same vertex set. A digraph $\mathcal{G}$ is weighted if a positive weight $\delta_{i j}$ is assigned to each arc $(i, j)$. Here $\delta_{i j}>0$ if and only if there exists an arc from vertex $j$ to vertex $i$ in $\mathcal{G}$, and we call $A=\left(\delta_{i j}\right)_{l \times l}$ the weight matrix. The weight $W(\mathcal{G})$ of $\mathcal{G}$ is the product of the weights on all its arcs. A directed path $\mathcal{P}$ in $\mathcal{G}$ is a subgraph with distinct vertices $\left\{i_{1}, i_{2}, \ldots, i_{s}\right\}$ such that its set of arcs is $\left\{\left(i_{k}, i_{k+1}\right): k=1,2, \ldots, s-1\right\}$. If $i_{s}=i_{1}$, we call $\mathcal{P}$ a directed cycle. A tree $\mathcal{T}$ is rooted at vertex $i$, called the root, if $i$ is not a terminal vertex of any arcs, and each of the remaining vertices is a terminal vertex of exactly one arc. A subgraph $\mathcal{Q}$ is unicyclic if it is a disjoint union of rooted trees whose roots form a directed cycle. A digraph $\mathcal{G}$ is strongly connected if for any pair of distinct vertices, there exists a directed path from one to the other. Denote the digraph with weight matrix $A$ as $(\mathcal{G}, A)$. A weighted digraph $(\mathcal{G}, A)$ is said to be balanced if $W(\mathcal{C})=W(-\mathcal{C})$ for all directed cycles $\mathcal{C}$. Here $-\mathcal{C}$ denotes the reverse of $\mathcal{C}$ and is constructed by reversing the direction of all arcs in $\mathcal{C}$. For a unicyclic graph $\mathcal{Q}$ with cycle $\mathcal{C}_{\mathcal{Q}}$, let $\tilde{\mathcal{Q}}$ be the unicyclic graph obtained by replacing $\mathcal{C}_{\mathcal{Q}}$ with $-\mathcal{C}_{\mathcal{Q}}$. Suppose that $(\mathcal{G}, A)$ is balanced, then $W(\mathcal{Q})=W(\tilde{\mathcal{Q}})$. The Laplacian matrix of $(\mathcal{G}, A)$ is defined as $L=\left(p_{k h}\right)_{l \times l}$, where $p_{k h}=-\delta_{k h}$ for $k \neq h$ and $p_{k h}=\sum_{j \neq k} \delta_{k j}$ for $k=h$. 